\title{
Role of hydrogen bonding in modifications of impact sensitivities of high energetic materials: evidence from crystal structures and quantum chemical calculations
}

\author{
D. Ž. Veljković ${ }^{1}$, D. S. Kretić1, I. S. Veljković², D. P. Malenov¹, D. B. Ninković로 S. D. Zarić1. \\ ${ }^{1}$ University of Belgrade - Faculty of Chemistry, Studentski trg, 12- 16, Belgrade, Serbia, ${ }^{2}$ University of Belgrade - Institute of \\ Chemistry, Technology and Metallurgy - National Institute of the Republic of Serbia, Njegoševa 12, Belgrade, Serbia, Innovation \\ center of the Faculty of Chemistry, Studentski trg 12-16, Belgrade, Serbia
}

vdusan@chem.bg.ac.rs

The development of new classes of high energetic materials (HEM) with high efficiency and low impact sensitivity is in the focus of numerous experimental and theoretical studies [1]. However, the high efficiency of HEM molecules is usually related to the high sensitivity towards detonation [2]. It is known that the sensitivity of HEM molecules towards detonation depends on many factors, including oxygen balance, energy content, and positive values of electrostatic potential above the central regions of the molecular surface. Analysis of positive values of molecular electrostatic potentials (MEP) showed to be an excellent tool in the assessment of impact sensitivities of high energetic molecules since positive values of MEP above the central regions of molecules are associated with high sensitivity towards detonation of HEM molecules [2]. Here we analysed the influence of hydrogen bonding on the values of the electrostatic potentials of fragments of HEM molecules extracted from crystal structures [3].

Crystal structures of three selected high energetic molecules were extracted from Cambridge Structural Database (CSD) and analysed in terms of non-covalent interactions. Three well-known HEM molecules were selected for the analysis: 1,3,5-Trinitrobenzene (TNB), 2,4,6-Trinitrophenol (TNP), and 2,4,6-Trinitrotoluene (TNT). Geometries of these molecules were used for electrostatic potentials calculations and for the design of model systems for interaction energies calculations. Electrostatic potential maps were calculated for TNB, TNP, and TNT geometries extracted from crystal structures for free molecules and molecules involved in hydrogen bonding. Values of electrostatic potentials above the central regions of molecules were analysed and compared for non-bonded HEM molecules and HEM molecules involved in hydrogen bonding.

Analysis of crystal structures showed that selected HEM molecules are involved in three types of hydrogen bonds: O-H...O-N interactions, $\mathrm{C}-\mathrm{H} \ldots \mathrm{O}-\mathrm{H}$ interactions, and in the case of TNP molecule $\mathrm{O}-\mathrm{H} \ldots \mathrm{O}-\mathrm{H}$ interactions. Analysis of positive values of the electrostatic potentials showed that hydrogen bonds have a significant influence on the values of the electrostatic potential in the central regions of HEM molecules. Calculations performed at M06/cc-PVDZ level showed that in the case when HEM molecules are involved in hydrogen bonding as hydrogen atom donors, positive values of electrostatic potentials in the centres of molecules decreased by $20-25 \%$. In the case when HEM molecules were involved in hydrogen bonding as hydrogen atom acceptors, positive values of electrostatic potentials in the centres of HEM molecules increased by $10 \%$.

Results presented in this study show that hydrogen bonds could be used as a tool for the modification of positive values of MEP above the central regions of HEM molecules and for the modification of their sensitivities towards detonation. Moderate change of positive electrostatic potential values above the central regions of HEM molecules upon formation of hydrogen bonds provide an opportunity for fine-tuning of sensitivities of HEM molecules towards detonation.

[1] Liu, G., Wei \& S.-H., Zhang, C., (2020). Cryst. Growth Des. 20, 7065.

[2] Politzer, P. \& Murray, J. S., (2015). J Mol Model, 21, 1.

[3] Kretić, D. S., Radovanović, J. I. \& Veljković, D. Ž., (2021). Phys. Chem. Chem. Phys., $23,7472$.

\section{Keywords: Cambridge Structural Database, quantum chemical calculations, high energetic materials, hydrogen bond}

This research was supported by the Science Fund of the Republic of Serbia, PROMIS, \#6066886, CD-HEM. This work was supported by the Serbian Ministry of Education, Science and Technological Development (Grant No. 451-03-9/2021-14/200026, 451-03-9/202114/200288 and 451-03-9/2021-14/200168). 